\title{
Chronic Toxicity Study of Dietary Stevia Extracts in F 344 Rats
}

\author{
(Received September 14, 1984)
}

\author{
Akio Yamada, Sumiko OhgaKı, Tsutomu Noda and Mitsuru Shimizu \\ (Osaka City Institute of Public Health and Environmental Sciences: \\ Tojo-cho 8-34, Tennoji-ku, Osaka, Japan)
}

\begin{abstract}
We fed 0 (control), $0.1,0.3$ and $1 \%$ stevia extracts (95.2\% purity) to $480 \mathrm{~F} 344$ rats for 22 months in the case of males and 24 months in the case of females. At 6 and 12 months, 10 animals of each sex from each group were sacrificed for clinical and pathological tests. The middle dose induced a slight growth retardation in both sexes, but at the high-dose the growth was reduced only transiently. General appearance and behavior were the same in all groups, including the control. Mortality at the end of the study in rats given stevia extracts was not significantly different from that in the controls. At 6 months, a variety of changes were found in the results of urinary, hematological and blood biochemical examinations and in organ weights, but there were no such differences at 12 months or at the end of the experiment. The incidence and severity of non-neoplastic and neoplastic changes were unrelated to the level of stevia extracts in the diet. The highest level of stevia extracts that causes no effects in rats was $550 \mathrm{mg} / \mathrm{kg}$, under the conditions of our experiments.
\end{abstract}

Key words: stevia extracts; chronic toxicity; carcinogenicity; dietary administration; F344 rat

\section{Introduction}

Leaves of Stevia rebaudiana BERTONI contain sweetening constituents such as stevioside and rebaudiosides $\mathrm{A}$ and $\mathrm{C}$. Stevia extracts have been used as a sweetener in Japan and elsewhere. Stevia crystals are 200 - to 300 -fold sweeter than sugar.

In mice, the oral median lethal dose of stevioside of $93.5 \%$ purity is more than $1.5 \mathrm{~g} / \mathrm{kg}$, and that of stevia extracts containing $53.1 \%$ stevioside is more than $4.2 \mathrm{~g} / \mathrm{kg} .{ }^{1)}$ When rats were given the extracts in their diet at dose levels of $0.28,1.40$ or $7.00 \%$ for 3 months, the highest dose caused a slight decrease in body weight gain in the males. ${ }^{1)}$ Planas and Kuć found that a decoction of Stevia rebaudiana reduced fertility in adult female rats. ${ }^{2)}$ According to Mori et al., however, administration of stevioside of $95.98 \%$ purity at dietary doses of $0.15,0.75$ or $3.00 \%$ to male rats for 60 days and to female rats for 14 days before mating caused no adverse effects on the pregnancy rate or the development of fetuses. ${ }^{3)}$

Rec-assay and reversion tests of crude ex- tracts of stevia containing about $18 \%$ stevioside, of purified extracts of stevia with about 55\% stevioside, and of stevia crystals, 95 98\% stevioside, were all negative. ${ }^{4)}$ Crude stevioside of $50 \%$ purity induced chromosomal aberrations in cultured Chinese hamster cells, but purified stevioside of $85 \%$ purity had no such effect. ${ }^{5), 8)}$

The purpose of this study was to investigate the chronic toxicity and carcinogenic potential of dietary stevia extracts in male and female F344 rats over about two years.

\section{Materials and Methods}

\section{Materials}

Hot water extracts from dry leaves of stevia were purified to a white crystalline powder. One hundred grams of the powder contained $95.20 \mathrm{~g}$ of total stevioside, consisting of $74.54 \mathrm{~g}$ of stevioside and $16.27 \mathrm{~g}$ of rebaudioside $\mathrm{A}$. The powder was supplied by Japan Stevia Association and stored at $4^{\circ} \mathrm{C}$ until use.

\section{Animals and their care}

Four-week-old F344/Du Crj rats of both sexes were purchased from Charles River Japan, Inc. Males weighing $108 \sim 135 \mathrm{~g}$ and females 
weighing $85 \sim 108 \mathrm{~g}$ after a ten-day acclimatization period were used.

One to three rats were housed in each stainless-steel wire cage; feed and tap water were provided ad libitum. The room temperature was $23 \pm 2{ }^{\circ} \mathrm{C}$ and the relative humidity was $60 \pm 20 \%$. Air in the room was changed more than fifteen times per hour.

Route of administration, doses and duration

The rats were fed $0.1,0.3$ or $1 \%$ stevia extracts in compressed chow (CRF-1, Charles River Japan, Inc.), which was freshly prepared at three-month intervals.

The highest dose in this test, $1 \%$ of the feed by weight, was decided based upon the annual consumption of sweeteners in Japan. Estimated consumption of sugar and isomerized sugar by Japanese was $25.92 \mathrm{~kg} /$ year or $71.0 \mathrm{~g} /$ day per person in 1980 . If stevia extracts were to account for a half of the total sweeteners by weight, the intake of stevia extracts would be about $4 \mathrm{mg} / \mathrm{kg} / \mathrm{day}$, because stevia extracts are 200 times as sweet as sugar, and the average body weight of Japanese is $50 \mathrm{~kg}$. When food intake in a mature rat was taken as about $50 \mathrm{~g} / \mathrm{kg} /$ day and the margin of safety was set at more than 100 , the highest dose to be used in this study could be calculated to be $1 \%$ of the total feed.

The 480 rats, 240 of each sex, used in this study were divided randomly into four groups: there were one control group, one middle-dose group, and one high-dose group, each containing 70 males and 70 females, and one low-dose group containing 30 males and 30 females.

The duration of the administration of stevia was 22 months for male rats and 24 months for females. At 6 and 12 months, 10 males and 10 females from each group were sacrificed for clinical and histopathological examinations.

Observations

At one-week intervals, individual body weights and food consumptions were recorded. Water intake was measured weekly for the first 48 weeks of the study and every four weeks thereafter.

All rats were checked daily. Every moribund animal found during the study was killed and tissue samples were taken for histopathological examination.

Urinalysis
At one or two weeks before sacrifice, $\mathrm{pH}$, protein, glucose, ketone bodies, occult blood and urobilinogen were measured using testpapers (Uro-Labstix, Miles-Sankyo Co., Ltd.).

\section{Hematological examinations}

At one week before sacrifice, blood from the tail vein was obtained. Erythrocyte and leucocyte counts (Coulter counter, Model ZBI, Coulter Electronics, Inc.), hemoglobin (cyanmethemoglobin method) and differential leucocyte counts were determined.

The animals were fasted for 24 hours before sacrifice. The blood was collected from the cervical vein just before sacrifice under light ether anesthesia. The hematocrit value was measured using microtubes and a centrifuge. Mean corpuscular volume (MCV), mean corpuscular hemoglobin $(\mathrm{MCH})$ and mean corpuscular hemoglobin concentration (MCHC) were calculated from the hematocrit and hemoglobin values and the erythrocyte counts.

\section{Blood biochemical examinations}

Serum from 10 rats of each sex from each group were obtained at 6 and 12 months for both sexes, and again at 22 months for males and at 24 months for females.

For each serum sample, the following determinations were performed: protein (using a refractometer), albumin (BCG method), GOT and GPT (UV one-point assay), alkaline phosphatase (ALP: Kind-King method), urea nitrogen (diacetyl-monoxime-thiosemicarbazide method), creatinine (Folin-Wu method), glucose (GODPOD method), triglycerides (GK-GPDH method), cholesterol (CHE-COD-POD method), sodium ion (with an ion electrode), potassium ion (with an ion electrode or by atomic absorption spectroscopy) and chlorine ion (SchalesChales method).

\section{Pathological examinations}

Some rats at 6 and 12 months, every moribund animal, and all surviving rats at the end of the test were sacrificed by exsanguination from the cervical vein under light ether anesthesia. All dead rats were autopsied as soon as they were found. The following organs were observed macroscopically: the liver, kidneys, spleen, lungs, heart, brain, pituitary, thymus, adrenal glands, prostate, testes, uterus and ovaries. These organs were removed and weighed, 
then microscopically observed together with the stomach, duodenum, jejunum, ileum, pancreas, bladder, bone marrow, thyroid and cervical lymph nodes. In addition, with the exception of the low-dose rats and the rats autopsied at 6 or 12 months, the following organs were observed microscopically: the vagina, tongue, esophagus, colon, cecum, rectum, submaxillary glands, skin, nasal cavity, spinal cord, larynx, mesenteric lymph nodes, external auditory canals, eyeballs, trachea, seminal vesicles, sternum, aorta and mammary glands.

The organs were fixed with buffered $10 \%$ formalin, and sections were stained with hematoxylin and eosin.

Statistical methods

For parameters expressed as means with standard error, statistical differences between the control and the groups given stevia extracts were examined by Yamazaki's ASSIT method ${ }^{8)}$ (based on Dunnett's ${ }^{9), 10)}$ and Scheffe's ${ }^{11}$ methods). Results of urinalysis other than $\mathrm{pH}$ and values expressed as percentages were analyzed by Wilcoxon's rank sum test. Urinary $\mathrm{pH}$ was statistically treated by the chi-square method.

\section{Results}

\section{Growth, food consumption and water intake}

Growth of the low-dose group was similar to that of the controls. In the middle-dose group, the rate of growth was slightly depressed after week 69 in males and after week 11 in females.
Transient growth retardation was observed in the high-dose group between week 23 and 50 in males and between week 60 and 75 in females. After week 69 in males and week 79 in females, the weights of rats tended to decrease in all groups, including the controls (Fig. 1).

Food consumption and water intake were higher in rats of the high-dose group than in the controls until week 35 .

The consumption of stevia extracts in the high-dose group was about $550 \mathrm{mg} / \mathrm{kg} / \mathrm{day}$ as calculated from the actual food intake during this experiment.

\section{General appearance}

Around week 5, wheezing was observed in some rats of either sex in all groups. Snivelling occurred in week 35 in some males and week 45 in some females.

A transient or persistent red ocular discharge, exophthalmos, and turbid cornea were seen in some males and females from every group.

Other abnormal signs found were as follows: subcutaneous and cutaneous solid masses in the back, shoulders, axillae, and abdomen, verrucae, focal pyogenic skin, alopecia, dirty hairs (especially at the hinder part of the abdomen), wry neck, ulcers and necrosis on the tail and loose stools. Piloerection, lacrimation, erection of the penis, and pale or yellowish skin and eyes were observed in some wasting rats. These signs occurred with almost equal frenquencies in all groups, including the controls.

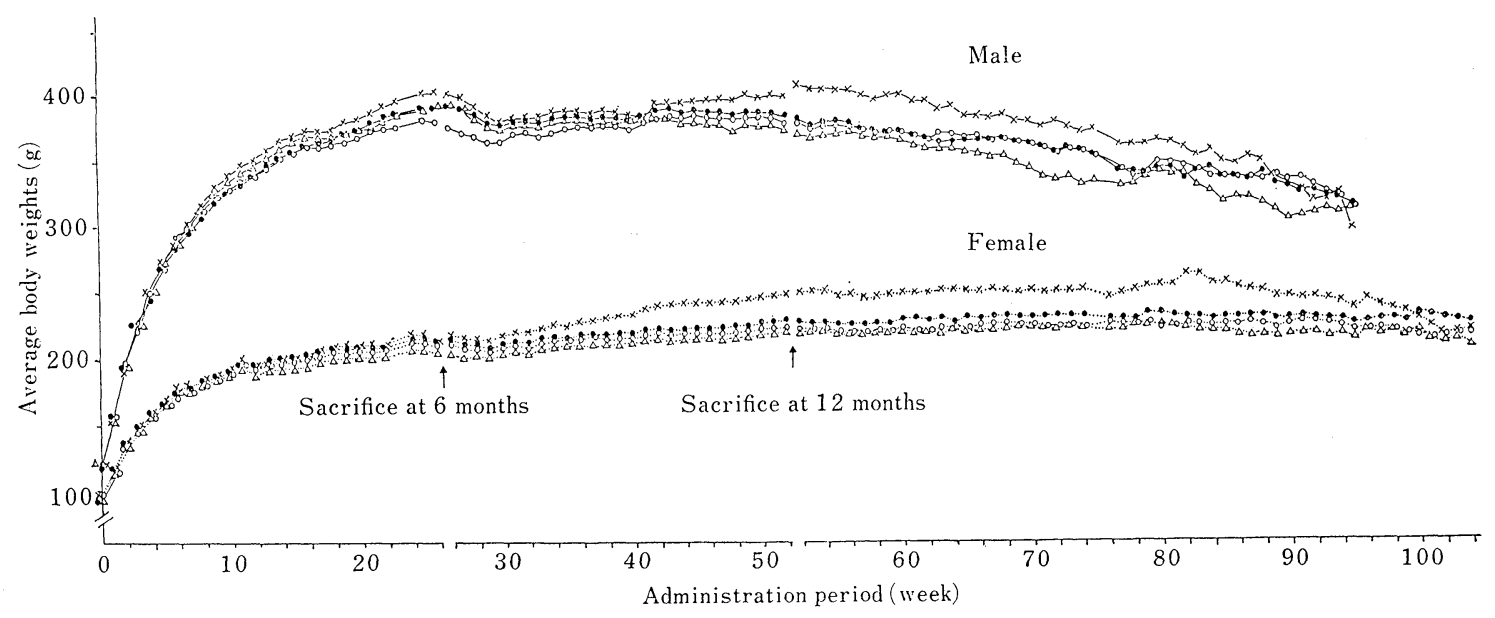

Fig. 1. Growth curves of rats given diets containing stevia extracts for 22 months (male) or 24 months (female): control $(\bullet), 0.1 \%$ in diet $(\times), 0.3 \%(\triangle)$ and $1 \%(O)$ 


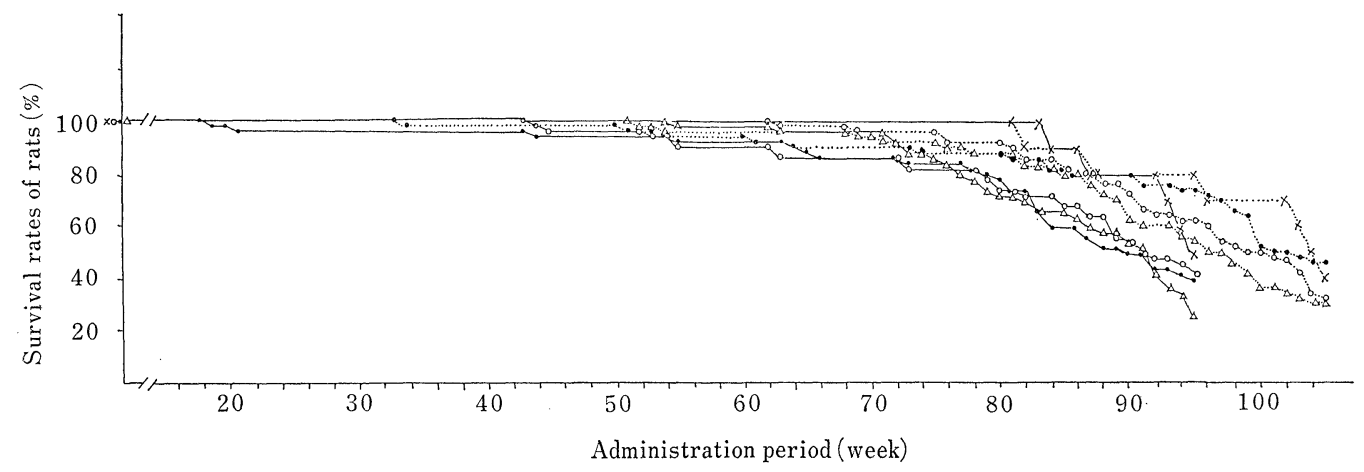

Fig. 2. Mortality of rats fed stevia extracts for 22 months (male; $\longrightarrow$ ) or for 24 months (female: - - - ) : control ( $)$, $0.1 \%$ in diet $(\times), 0.3 \%(\triangle)$, and $1 \%(\bigcirc)$

Fig. 2 shows the mortality data. After the 90th week, the mortalities of middle-dose males and of middle- and high-dose females tended to be higher than that of the controls, but the differences were not statistically significant.

Urinalysis, hematological and blood biochemical examinations

In the urinalysis, no dose-related changes were found, except for an increased incidence of proteinuria in females at 6 months.

Dose-related increases in erythrocyte counts and decreases in $\mathrm{MCV}$ and $\mathrm{MCH}$ in male rats, and decreases in erythrocyte counts in female rats were found at 6 months, but when the results of hematological examinations of the controls and the groups fed stevia extracts at 12 months and at the end of the study were statistically analyzed, the differences were not signif- icant (Tables 1 4).

Administration of stevia extracts for 6 months caused an increase of blood glucose in both sexes and decreases of GOT, GPT and triglycerides in the females. There were, however, no significant differences in blood biochemical findings at 12 months or at the end of the experiment (Tables 5 8).

Organ weights and macroscopic observations

In the high-dose group at 6 months, the absolute weights of the liver, kidneys, heart, prostate and testes in males were significantly increased and those of the ovaries in females were significantly decreased, whereas the relative weight of the liver in females was increased. These changes were not found in either sex at 12 months or at the end of the study (Tables $9 \sim 12$ ).

Table 1. Hematological Values of Male Rats Fed Stevia Extracts for 6 Months

\begin{tabular}{|c|c|c|c|c|c|c|c|}
\hline Group & No. & $\begin{array}{c}\mathrm{RBC} \\
\times 10^{4} / \mathrm{mm}^{3}\end{array}$ & $\begin{array}{c}\text { Hemaglobin } \\
\text { (g/dl) }\end{array}$ & $\begin{array}{c}\text { Hematocrit } \\
(\%)\end{array}$ & $\begin{array}{l}\mathrm{MCV} \\
\left(\mu \mathrm{m}^{3}\right)\end{array}$ & $\begin{array}{c}\mathrm{MCH} \\
(\mathrm{pg})\end{array}$ & $\begin{array}{c}\text { MCHC } \\
(\%)\end{array}$ \\
\hline Control & 10 & $865 \pm 18.5$ & $16.8 \pm 0.24$ & $51.9 \pm 0.42$ & $60.3 \pm 1.64$ & $19.5 \pm 0.51$ & $32.4 \pm 0.47$ \\
\hline Low-dose & 10 & $892 \pm 15.9$ & $16.0 \pm 0.21$ & $51.7 \pm 0.31$ & $58.1 \pm 0.85$ & $18.0 \pm 0.20 *$ & $31.0 \pm 0.33$ \\
\hline Middle-dose & 10 & $887 \pm 8.7$ & $16.2 \pm 0.22$ & $51.3 \pm 0.50$ & $57.9 \pm 0.60$ & $18.2 \pm 0.11$ & $31.6 \pm 0.44$ \\
\hline High-dose & 10 & $946 \pm 15.4^{* *}$ & $16.8 \pm 0.30$ & $51.5 \pm 0.38$ & $54.5 \pm 0.96^{* *}$ & $17.8 \pm 0.10^{* *}$ & $32.7 \pm 0.62$ \\
\hline \multirow{2}{*}{ Group } & \multirow{2}{*}{ No. } & WBC & \multicolumn{5}{|c|}{ Differential leucocyte count $(\%)$} \\
\hline & & $\times 10^{2} / \mathrm{mm}^{3}$ & Baso. & Eosin. & Neut. & Lym. & Mono. \\
\hline Control & 10 & $151 \pm 9.8$ & 0.0 & $1.8 \pm 0.44$ & $19.3 \pm 1.54$ & $78.3 \pm 1.54$ & $0.6 \pm 0.22$ \\
\hline Low-dose & 10 & $149 \pm 6.4$ & 0.0 & $1.9 \pm 0.46$ & $21.2 \pm 1.38$ & $76.2 \pm 1.69$ & $0.7 \pm 0.26$ \\
\hline Middle-dose & 10 & $163 \pm 10.4$ & 0.0 & $2.4 \pm 0.45$ & $18.9 \pm 1.24$ & $78.0 \pm 1.46$ & $0.7 \pm 0.33$ \\
\hline High-dose & 10 & $164 \pm 12.0$ & 0.0 & $1.7 \pm 0.21$ & $21.7 \pm 1.13$ & $75.9 \pm 1.02$ & $0.7 \pm 0.33$ \\
\hline
\end{tabular}

Mean \pm standard error

Significantly different from the control at $* \mathrm{P}<0.05$ or $* * \mathrm{P}<0.01$ 
Table 2. Hematological Values of Female Rats Fed Stevia Extracts for 6 Months

\begin{tabular}{|c|c|c|c|c|c|c|c|}
\hline Group & No. & $\begin{array}{c}\mathrm{RBC} \\
\times 10^{4} / \mathrm{mm}^{3}\end{array}$ & $\begin{array}{l}\text { Hemoglobin } \\
\text { (g/dl) }\end{array}$ & $\begin{array}{c}\text { Hematocrit } \\
(\%)\end{array}$ & $\begin{array}{l}\mathrm{MCV} \\
\left(\mu \mathrm{m}^{3}\right)\end{array}$ & $\begin{array}{l}\mathrm{MCH} \\
(\mathrm{pg})\end{array}$ & $\begin{array}{c}\mathrm{MCHC} \\
(\%)\end{array}$ \\
\hline Control & 10 & $845 \pm 6.3$ & $16.2 \pm 0.20$ & $51.5 \pm 0.29$ & $61.0 \pm 0.62$ & $19.2 \pm 0.30$ & $31.5 \pm 0.48$ \\
\hline Low-dose & 10 & $804 \pm 12.9$ & $15.6 \pm 0.26$ & $52.2 \pm 0.47$ & $65.1 \pm 1.15$ & $19.5 \pm 0.14$ & $30.0 \pm 0.57$ \\
\hline Middle-dose & 10 & $799 \pm 24.0$ & $15.7 \pm 0.38$ & $50.3 \pm 0.23$ & $63.5 \pm 1.94$ & $19.7 \pm 0.22$ & $31.3 \pm 0.78$ \\
\hline High-dose & 10 & $782 \pm 16.4^{*}$ & $16.5 \pm 0.41$ & $50.3 \pm 0.48$ & $64.5 \pm 1.53$ & $21.1 \pm 0.59$ & $32.8 \pm 0.81$ \\
\hline \multirow{2}{*}{ Group } & \multirow{2}{*}{ No. } & WBC & \multicolumn{5}{|c|}{ Differential leucocyte count (\%) } \\
\hline & & $\times 10^{2} / \mathrm{mm}^{3}$ & Baso. & Eosin. & Neut. & Lym. & Mono. \\
\hline Control & 10 & $134 \pm 8.1$ & 0.0 & $1.6 \pm 0.50$ & $17.0 \pm 2.18$ & $80.7 \pm 2.17$ & $0.7 \pm 0.26$ \\
\hline Low-dose & 10 & $132 \pm 8.1$ & 0.0 & $1.9 \pm 0.41$ & $18.4 \pm 2.23$ & $78.9 \pm 2.18$ & $0.8 \pm 0.25$ \\
\hline Middle-dose & 10 & $113 \pm 7.3$ & 0.0 & $1.5 \pm 0.40$ & $19.2 \pm 1.76$ & $78.8 \pm 2.06$ & $0.5 \pm 0.22$ \\
\hline High-dose & 10 & $116 \pm 5.9$ & 0.0 & $1.7 \pm 0.37$ & $16.0 \pm 1.32$ & $81.5 \pm 1.34$ & $0.8 \pm 0.25$ \\
\hline
\end{tabular}

Mean \pm standard error

Significantly different from the control at $* \mathrm{P}<0.05$

Table 3. Hematological Values of Male Rats Fed Stevia Extracts for 22 Months

\begin{tabular}{|c|c|c|c|c|c|c|c|}
\hline Group & No. & $\begin{array}{c}\mathrm{RBC} \\
\times 10^{4} / \mathrm{mm}^{3}\end{array}$ & $\begin{array}{l}\text { Hemoglobin } \\
\qquad(\mathrm{g} / \mathrm{dl})\end{array}$ & $\begin{array}{c}\text { Hematocrit } \\
(\%)\end{array}$ & $\begin{array}{l}\mathrm{MCV} \\
\left(\mu \mathrm{m}^{3}\right)\end{array}$ & $\begin{array}{r}\mathrm{MCH} \\
(\mathrm{pg})\end{array}$ & $\begin{array}{c}\mathrm{MCHC} \\
(\%)\end{array}$ \\
\hline Control & 20 & $932 \pm 18.4$ & $21.2 \pm 0.54$ & $60.0 \pm 1.16$ & $64.6 \pm 1.16$ & $22.7 \pm 0.29$ & $35.4 \pm 0.81$ \\
\hline Low-dose & 5 & $857 \pm 62.1$ & $19.2 \pm 1.58$ & $52.9 \pm 7.69$ & $60.2 \pm 5.94$ & $22.4 \pm 0.76$ & $39.3 \pm 5.43$ \\
\hline Middle-dose & 13 & $851 \pm 42.6$ & $18.9 \pm 0.78$ & $53.1 \pm 3.54$ & $62.5 \pm 3.26$ & $22.6 \pm 1.01$ & $37.2 \pm 2.35$ \\
\hline High-dose & 21 & $921 \pm 24.0$ & $20.1 \pm 0.66$ & $55.3 \pm 2.59$ & $59.9 \pm 2.16$ & $21.8 \pm 0.37$ & $37.3 \pm 1.25$ \\
\hline \multirow{2}{*}{ Group } & \multirow{2}{*}{ No. } & WBC & \multicolumn{5}{|c|}{ Differential leucocyte count $(\%)$} \\
\hline & & $\times 10^{2} / \mathrm{mm}^{3}$ & Baso. & Eosin. & Neut. & Lym. & Mono. \\
\hline Control & 20 & $190 \pm 9.9$ & $0.1 \pm 0.05$ & $1.6 \pm 0.19$ & $38.4 \pm 2.08$ & $59.2 \pm 2.09$ & $0.7 \pm 0.12$ \\
\hline Low-dose & 5 & $213 \pm 29.9$ & $0.0 \pm 0.0$ & $1.9 \pm 0.51$ & $41.9 \pm 1.91$ & $55.4 \pm 1.91$ & $0.8 \pm 0.34$ \\
\hline Middle-dose & 13 & $320 \pm 78.5$ & $0.0 \pm 0.04$ & $2.2 \pm 0.45$ & $36.0 \pm 2.84$ & $61.3 \pm 3.07$ & $0.5 \pm 0.11$ \\
\hline High-dose & 21 & $255 \pm 21.6$ & $0.1 \pm 0.06$ & $2.0 \pm 0.29$ & $33.6 \pm 2.15$ & $63.6 \pm 2.26$ & $0.7 \pm 0.11$ \\
\hline
\end{tabular}

Mean \pm standard error

Table 4. Hematological Values of Female Rats Fed Stevia Extracts for 24 Months

\begin{tabular}{|c|c|c|c|c|c|c|c|}
\hline Group & No. & $\begin{array}{c}\mathrm{RBC} \\
\times 10^{4} / \mathrm{mm}^{3} \\
\end{array}$ & $\begin{array}{c}\text { Hemoglobin } \\
(\mathrm{g} / \mathrm{dl})\end{array}$ & $\begin{array}{c}\text { Hematocrit } \\
(\%)\end{array}$ & $\begin{array}{l}\mathrm{MCV} \\
\left(\mu \mathrm{m}^{3}\right)\end{array}$ & $\begin{array}{l}\mathrm{MCH} \\
(\mathrm{pg})\end{array}$ & $\begin{array}{c}\mathrm{MCHC} \\
(\%)\end{array}$ \\
\hline Control & 23 & $781 \pm 29.5$ & $17.1 \pm 0.40$ & $51.5 \pm 1.69$ & $67.5 \pm 3.11$ & $22.3 \pm 0.63$ & $33.6 \pm 0.98$ \\
\hline Low-dose & 4 & $877 \pm 41.5$ & $18.6 \pm 1.34$ & $50.1 \pm 1.13$ & $57.6 \pm 3.37$ & $21.1 \pm 0.50$ & $37.2 \pm 3.37$ \\
\hline Middle-dose & 15 & $757 \pm 42.2$ & $17.4 \pm 0.69$ & $50.9 \pm 2.79$ & $71.3 \pm 6.81$ & $22.0 \pm 0.60$ & $35.0 \pm 2.52$ \\
\hline High-dose & 17 & $826 \pm 29.6$ & $17.4 \pm 0.55$ & $46.3 \pm 2.83$ & $57.4 \pm 3.64$ & $21.2 \pm 0.41$ & $40.0 \pm 3.50$ \\
\hline \multirow{2}{*}{ Group } & \multirow{2}{*}{ No. } & WBC & \multicolumn{5}{|c|}{ Differential leucocyte count $(\%)$} \\
\hline & & $\times 10^{2} / \mathrm{mm}^{3}$ & Baso. & Eosin. & Neut. & Lym. & Mono. \\
\hline Control & 23 & $189 \pm 13.5$ & $0.1 \pm 0.04$ & $1.3 \pm 0.21$ & $26.0 \pm 1.72$ & $72.1 \pm 1.75$ & $0.5 \pm 0.12$ \\
\hline Low-dose & 4 & $170 \pm 22.5$ & $0.1 \pm 0.13$ & $4.3 \pm 3.10$ & $32.0 \pm 5.43$ & $62.8 \pm 4.15$ & $0.9 \pm 0.24$ \\
\hline Middle-dose & 15 & $448 \pm 253.5$ & $0.0 \pm 0.03$ & $1.4 \pm 0.17$ & $28.2 \pm 2.07$ & $69.7 \pm 2.16$ & $0.7 \pm 0.19$ \\
\hline High-dose & 17 & $158 \pm 12.5$ & $0.0 \pm 0.03$ & $1.7 \pm 0.36$ & $28.8 \pm 2.50$ & $69.0 \pm 2.37$ & $0.5 \pm 0.12$ \\
\hline
\end{tabular}

Mean \pm standard error 
Table 5. Blood Biochemistry Values of Male Rats Fed Stevia Extracts for 6 Months

\begin{tabular}{|c|c|c|c|c|c|c|c|c|}
\hline Group & No. & $\begin{array}{l}\text { Protein } \\
(\mathrm{g} / \mathrm{dl})\end{array}$ & $\mathrm{A} / \mathrm{G}$ & $\begin{array}{l}\text { GOT } \\
\text { K-unit }\end{array}$ & $\begin{array}{l}\mathrm{GP} \\
\mathrm{K}-\mathrm{u}\end{array}$ & unit & $\begin{array}{c}\text { ALP } \\
\text { KA-unit }\end{array}$ & $\begin{array}{l}\text { Urea } N \\
(\mathrm{mg} / \mathrm{dl})\end{array}$ \\
\hline Control & 10 & $7.0 \pm 0.06$ & $1.19 \pm 0.025$ & $117 \pm 5.4$ & $64.7 \pm$ & $=3.23$ & $10.8 \pm 0.80$ & $17.2 \pm 0.54$ \\
\hline Low-dose & 10 & $7.0 \pm 0.04$ & $1.36 \pm 0.056^{*}$ & $118 \pm 10.8$ & $82.3 \pm$ & $=16.82$ & $10.1 \pm 0.40$ & $16.9 \pm 0.37$ \\
\hline Middle-dose & 10 & $7.1 \pm 0.05$ & $1.22 \pm 0.041$ & $104 \pm 9.6$ & $64.1 \pm$ & $=8.56$ & $10.8 \pm 0.58$ & $16.9 \pm 0.36$ \\
\hline High-dose & 10 & $6.9 \pm 0.06$ & $1.27 \pm 0.036$ & $94 \pm 5.3$ & $56.3 \pm$ & 2.74 & $10.3 \pm 0.40$ & $17.8 \pm 0.47$ \\
\hline Group & No. & $\begin{array}{l}\text { Creatinine } \\
(\mathrm{mg} / \mathrm{dl})\end{array}$ & $\begin{array}{l}\text { Glucose } \\
(\mathrm{mg} / \mathrm{dl})\end{array}$ & $\begin{array}{l}\text { Triglyce- } \\
\text { rides } \\
(\mathrm{mg} / \mathrm{dl})\end{array}$ & $\begin{array}{l}\text { Choles- } \\
\text { terol } \\
(\mathrm{mg} / \mathrm{dl})\end{array}$ & $\begin{array}{c}\mathrm{Na}^{+} \\
(\mathrm{mEq} / \mathrm{I}\end{array}$ & $\begin{array}{c}\mathrm{K}^{+} \\
(\mathrm{mEq} / \mathrm{L})\end{array}$ & $\begin{array}{c}\mathrm{Cl}^{-} \\
(\mathrm{mEq} / \mathrm{L})\end{array}$ \\
\hline Control & 10 & $0.81 \pm 0.019$ & $111 \pm 3.5$ & $133 \pm 10.5$ & $56 \pm 2.0$ & $145 \pm 1$. & $3 \quad 4.7 \pm 0.10$ & $101 \pm 0.3$ \\
\hline Low-dose & 10 & $0.80 \pm 0.015$ & $122 \pm 4.2$ & $146 \pm 10.2$ & $63 \pm 2.1$ & $141 \pm 1$. & $0 \quad 4.4 \pm 0.05$ & $102 \pm 0.6$ \\
\hline Middle-dose & 10 & $0.81 \pm 0.013$ & $128 \pm 3.2 * *$ & $114 \pm 16.8$ & $58 \pm 4.4$ & $142 \pm 1$ & $0 \quad 4.4 \pm 0.08^{*}$ & $102 \pm 0.5$ \\
\hline High-dose & 10 & $0.80 \pm 0.017$ & $133 \pm 3.7 * *$ & $110 \pm 20.9$ & $57 \pm 3.5$ & $143 \pm 0$. & $8 \quad 4.5 \pm 0.07$ & $101 \pm 0.5$ \\
\hline
\end{tabular}

Mean \pm standard error

Significantly different from the control at $* \mathrm{P}<0.05$ or $* * \mathrm{P}<0.01$

Table 6. Blood Biochemistry Values of Female Rats Fed Stevia Extracts for 6 Months

\begin{tabular}{|c|c|c|c|c|c|c|c|c|}
\hline Group & No. & $\begin{array}{l}\text { Protein } \\
(\mathrm{g} / \mathrm{dl})\end{array}$ & $\mathrm{A} / \mathrm{G}$ & $\begin{array}{c}\text { GOT } \\
\text { K-unit }\end{array}$ & $\begin{array}{c}\text { GPT } \\
\text { K-unit }\end{array}$ & \multicolumn{2}{|c|}{$\begin{array}{c}\text { ALP } \\
\text { KA-unit }\end{array}$} & $\begin{array}{l}\text { Urea } N \\
(\mathrm{mg} / \mathrm{dl})\end{array}$ \\
\hline Control & 10 & $7.4 \pm 0.12$ & $1.34 \pm 0.092$ & $108 \pm 6.5$ & $51.9 \pm 2.48$ & \multicolumn{2}{|c|}{$11.7 \pm 0.89$} & $19.6 \pm 0.35$ \\
\hline Low-dose & 10 & $7.6 \pm 0.07$ & $1.25 \pm 0.035$ & $118 \pm 7.0$ & $57.6 \pm 3.06$ & \multicolumn{2}{|c|}{$11.1 \pm 0.69$} & $18.5 \pm 0.29$ \\
\hline Middle-dose & 10 & $7.3 \pm 0.07$ & $1.39 \pm 0.037$ & $102 \pm 4.2$ & $48.8 \pm 2.80$ & \multicolumn{2}{|c|}{$8.5 \pm 0.38^{* *}$} & $18.7 \pm 0.47$ \\
\hline High-dose & 10 & $7.3 \pm 0.07$ & $1.30 \pm 0.075$ & $91 \pm 2.5^{*}$ & $36.4 \pm 2.22 *$ & \multicolumn{2}{|c|}{$10.7 \pm 0.67$} & $19.2 \pm 0.38$ \\
\hline & & $\begin{array}{l}\text { Creatinine } \\
(\mathrm{mg} / \mathrm{dl})\end{array}$ & $\begin{array}{l}\text { Glucose } \\
(\mathrm{mg} / \mathrm{dl})\end{array}$ & $\begin{array}{l}\text { Triglyce- } \\
\text { rides } \\
(\mathrm{mg} / \mathrm{dl})\end{array}$ & $\begin{array}{l}\text { Choles- } \\
\text { terol } \\
(\mathrm{mg} / \mathrm{dl})\end{array}$ & $\begin{array}{c}\mathrm{Na}^{+} \\
(\mathrm{mEq} / \mathrm{L})\end{array}$ & $\begin{array}{c}\mathrm{K}^{+} \\
(\mathrm{mEq} / \mathrm{L})\end{array}$ & $\begin{array}{c}\mathrm{Cl}^{-} \\
(\mathrm{mEq} / \mathrm{L})\end{array}$ \\
\hline Control & 10 & $0.83 \pm 0.023$ & $88 \pm 5.4$ & $65 \pm 2.6$ & $61 \pm 3.5$ & $140 \pm 1.1$ & $4.0 \pm 0.12$ & $106 \pm 0.5$ \\
\hline Low-dose & 10 & $0.82 \pm 0.015$ & $97 \pm 2.9$ & $58 \pm 3.3$ & $62 \pm 2.5$ & $143 \pm 1.0$ & $4.1 \pm 0.08$ & $106 \pm 0.7$ \\
\hline Middle-dose & 10 & $0.83 \pm 0.018$ & $103 \pm 3.5^{*}$ & $53 \pm 3.0^{*}$ & $67 \pm 3.8$ & $139 \pm 1.2$ & $4.0 \pm 0.05$ & $106 \pm 0.8$ \\
\hline High-dose & 10 & $0.85 \pm 0.022$ & $101 \pm 2.8$ & $44 \pm 1.8 * *$ & $62 \pm 3.1$ & $142 \pm 1.5$ & $4.1 \pm 0.07$ & $107 \pm 0.4$ \\
\hline
\end{tabular}

Mean \pm standard error

Significantly different from the control at $* \mathrm{P}<0.05$ or $* * \mathrm{P}<0.01$

Table 7. Blood Biochemistry Values of Male Rats Fed Stevia Extracts for 22 Months

\begin{tabular}{|c|c|c|c|c|c|c|c|c|c|}
\hline Group & No. & $\begin{array}{l}\text { Protein } \\
(\mathrm{g} / \mathrm{dl})\end{array}$ & $\mathrm{A} / \mathrm{G}$ & $\begin{array}{l}\text { GOT } \\
\text { K-unit }\end{array}$ & \multicolumn{2}{|c|}{$\begin{array}{c}\text { GPT } \\
\text { K-unit }\end{array}$} & \multicolumn{2}{|c|}{$\begin{array}{c}\text { ALP } \\
\text { KA-unit }\end{array}$} & $\begin{array}{l}\text { Urea N } \\
(\mathrm{mg} / \mathrm{dl})\end{array}$ \\
\hline Control & 10 & $6.8 \pm 0.22$ & $1.01 \pm 0.062$ & $142 \pm 25.5$ & $93.2 \pm 28$ & 8.58 & 26. & $5 \pm 5.13$ & $22.4 \pm 2.24$ \\
\hline Low-dose & 5 & $6.4 \pm 0.13$ & $0.95 \pm 0.052$ & $126 \pm 15.7$ & $68.5 \pm 7$ & 7.30 & 40. & $8 \pm 7.35$ & $44.0 \pm 17.46$ \\
\hline Middle-dose & 10 & $7.0 \pm 0.15$ & $0.93 \pm 0.041$ & $134 \pm 13.2$ & $65.7 \pm$ & 9.49 & 20. & $6 \pm 3.66$ & $26.0 \pm 2.16$ \\
\hline High-dose & 10 & $6.7 \pm 0.17$ & $1.08 \pm 0.019$ & $171 \pm 25.4$ & $78.1 \pm 16$ & 6.57 & 21 & $5 \pm 2.16$ & $24.9 \pm 2.57$ \\
\hline Group & No. & $\begin{array}{l}\text { Creatinine } \\
(\mathrm{mg} / \mathrm{dl})\end{array}$ & $\begin{array}{l}\text { Glucose } \\
(\mathrm{mg} / \mathrm{dl})\end{array}$ & $\begin{array}{c}\text { Triglyce- } \\
\text { rides } \\
(\mathrm{mg} / \mathrm{dl})\end{array}$ & $\begin{array}{c}\text { Choles- } \\
\text { terol } \\
(\mathrm{mg} / \mathrm{dl})\end{array}$ & $\begin{array}{r}\mathrm{Na} \\
(\mathrm{mEC}\end{array}$ & & $\begin{array}{c}\mathrm{K}^{+} \\
(\mathrm{mEq} / \mathrm{L})\end{array}$ & $\begin{array}{c}\mathrm{Cl}^{-} \\
(\mathrm{mEq} / \mathrm{L})\end{array}$ \\
\hline Control & 10 & $0.90 \pm 0.061$ & $68 \pm 4.0$ & $175 \pm 24.9$ & $153 \pm 15.7$ & $145 \pm$ & 0.7 & $4.5 \pm 0.21$ & $102 \pm 0.8$ \\
\hline Low-dose & 5 & $0.92 \pm 0.164$ & $68 \pm 13.3$ & $126 \pm 30.7$ & $143 \pm 15.5$ & $153 \pm$ & 4.1 & $5.1 \pm 0.29$ & $99 \pm 1.7$ \\
\hline Middle-dose & 10 & $0.84 \pm 0.060$ & $68 \pm 7.8$ & $274 \pm 53.3$ & $225 \pm 32.1$ & $144 \pm$ & 1.1 & $4.6 \pm 0.17$ & $99 \pm 0.9$ \\
\hline High-dose & 10 & $0.82 \pm 0.063$ & $63 \pm 7.0$ & $159 \pm 22.6$ & $140 \pm 10.9$ & $144 \pm$ & 2.8 & $4.8 \pm 0.18$ & $103 \pm 1.6$ \\
\hline
\end{tabular}

Mean \pm standard error 
Table 8. Blood Biochemistry Values of Female Rats Fed Stevia Extract for 24 Months

\begin{tabular}{|c|c|c|c|c|c|c|c|c|c|}
\hline Group & No. & $\begin{array}{l}\text { Protein } \\
(\mathrm{g} / \mathrm{dl})\end{array}$ & $\mathrm{A} / \mathrm{G}$ & $\begin{array}{l}\text { GOT } \\
\text { K-unit }\end{array}$ & \multicolumn{2}{|c|}{$\begin{array}{c}\text { GPT } \\
\text { K-unit }\end{array}$} & \multicolumn{2}{|c|}{$\begin{array}{c}\text { ALP } \\
\text { KA-unit }\end{array}$} & $\begin{array}{l}\text { Urea N } \\
(\mathrm{mg} / \mathrm{dl})\end{array}$ \\
\hline Control & 10 & $6.6 \pm 0.30$ & $0.98 \pm 0.055$ & $152 \pm 30.3$ & $81.6 \pm 27$ & .18 & 29.7 & \pm 3.34 & $21.1 \pm 4.28$ \\
\hline Low-dose & 4 & $7.0 \pm 0.26$ & $0.94 \pm 0.044$ & $124 \pm 32.1$ & $83.9 \pm 26$ & 5.16 & 24.2 & \pm 4.34 & $18.0 \pm 0.69$ \\
\hline Middle-dose & 10 & $7.1 \pm 0.26$ & $0.85 \pm 0.056$ & $115 \pm 10.3$ & $60.5 \pm 4$ & 72 & 33.3 & \pm 7.58 & $34.3 \pm 13.56$ \\
\hline High-dose & 10 & $6.7 \pm 0.24$ & $0.94 \pm 0.063$ & $104 \pm 11.6$ & $56.5 \pm 6$ & .62 & 24.9 & \pm 2.54 & $21.1 \pm 2.43$ \\
\hline Group & No. & $\begin{array}{l}\text { Creatinine } \\
(\mathrm{mg} / \mathrm{dl})\end{array}$ & $\begin{array}{l}\text { Glucose } \\
(\mathrm{mg} / \mathrm{dl})\end{array}$ & $\begin{array}{c}\text { Triglyce- } \\
\text { rides } \\
(\mathrm{mg} / \mathrm{dl})\end{array}$ & $\begin{array}{c}\text { Choles- } \\
\text { terol } \\
(\mathrm{mg} / \mathrm{dl})\end{array}$ & $\begin{array}{r}1 \\
(\mathrm{mF} \\
\end{array}$ & $\begin{array}{l}a^{+} \\
q / L)\end{array}$ & $\begin{array}{c}\mathrm{K}^{+} \\
(\mathrm{mEq} / \mathrm{L}) \\
\end{array}$ & $\begin{array}{c}\mathrm{Cl}^{-} \\
(\mathrm{mEq} / \mathrm{L})\end{array}$ \\
\hline Control & 10 & $0.86 \pm 0.091$ & $60 \pm 8.9$ & $113 \pm 14.2$ & $104 \pm 14.9$ & 146 & \pm 1.3 & $4.6 \pm 0.54$ & $34 \quad 100 \pm 0.5$ \\
\hline Low-dose & 4 & $0.80 \pm 0.042$ & $64 \pm 12.2$ & $106 \pm 20.0$ & $135 \pm 12.9$ & 141 & \pm 2.6 & $4.3 \pm 0.15$ & $.5101 \pm 0.5$ \\
\hline Middle-dose & 10 & $0.85 \pm 0.055$ & $45 \pm 7.8$ & $132 \pm 20.7$ & $163 \pm 41.0$ & 143 & \pm 1.2 & $4.6 \pm 0.15$ & $99 \pm 1.2$ \\
\hline High-dose & 10 & $0.81 \pm 0.034$ & $62 \pm 7.2$ & $137 \pm 35.4$ & $145 \pm 30.3$ & 140 & \pm 3.4 & $4.2 \pm 0.10$ & $99 \pm 1.2$ \\
\hline
\end{tabular}

Mean \pm standard error

In some rats killed at 6 months from every group, there were small nodules in the liver (about 3 9 $\mathrm{mm}$ in diameter) and reddish-brown areas in the lungs. At 12 months, small nodules of the liver, hepatization of the lungs, focal or diffuse reddish-brown areas in the lungs, enlargement of the pituitary, hemorrhage of the thymus, enlargement of the cervical lymph nodes, and edema with hemorrhage of the uterus were observed in rats of all groups. These changes were not related to dose.

With longer feeding of stevia extracts, small nodules, discoloration and a dull margin on the liver, hepatization and abscess of the lungs, and a granular surface of the kidneys were found in many rats in each group. The testes of many male rats were reddish-purple with a brown tinge, and those of more than half of the rats were enlarged or atrophied. Other findings in all groups were as follows: enlargement, hemorrhage and hematocele of the pituitary: hemorrhage of the stomach; enlargement of the spleen (except in males); enlargement of the prostate; thickness, hemorrhage and edema of the uterus and enlargement of the ovaries. Enlargement of the spleen in male rats was seen only in those given stevia extracts.

\section{Histopathological findings}

Peribronchitis was observed in many rats autopsied at 6 months. Bronchitis and peribronchitis were found in all rats autopsied at 12 months. The kidneys in some rats of every group had hyaline casts, lymphocyte infiltration in the cortex and vacuolar degeneration in the tubules.

For rats other than those sacrificed at 6 and 12 months, non-neoplastic changes are listed in Table 13. In most cases, the groups fed stevia extracts and the controls had similar incidences of these changes. Changes more common in rats given stevia extracts were reduced spermatogenesis, interstitial cell proliferation in the testes, medullary cell proliferation in the adrenal glands, atrophy of the thymus, inflammatory lesions in the trachea and lungs, age-related changes of the kidneys such as degeneration of tubular epithelium, hyaline casts and glomerular sclerosis, and pigmentation and increased hematogenesis of the spleen.

Tables 14 and 15 list all neoplasms found. Organs in the low-dose group (shown as - in the tables) were not inspected microscopically. The most common neoplasms in the males were interstitial cell adenoma of the testes and pheochromocytoma of the adrenal glands. In the females, they were interstitial polyps of the endometrium and adenoma of the anterior lobe of the pituitary. The incidence of neoplasms was not related to dose.

\section{Discussion}

Food additives are incorporated daily into the human body via foods throughout life. Many kinds of cancer have a long latency, appearing only in old age. Therefore, the duration of chronic toxicity and carcinogenicity tests of food additives in rats is usually 24 months or 


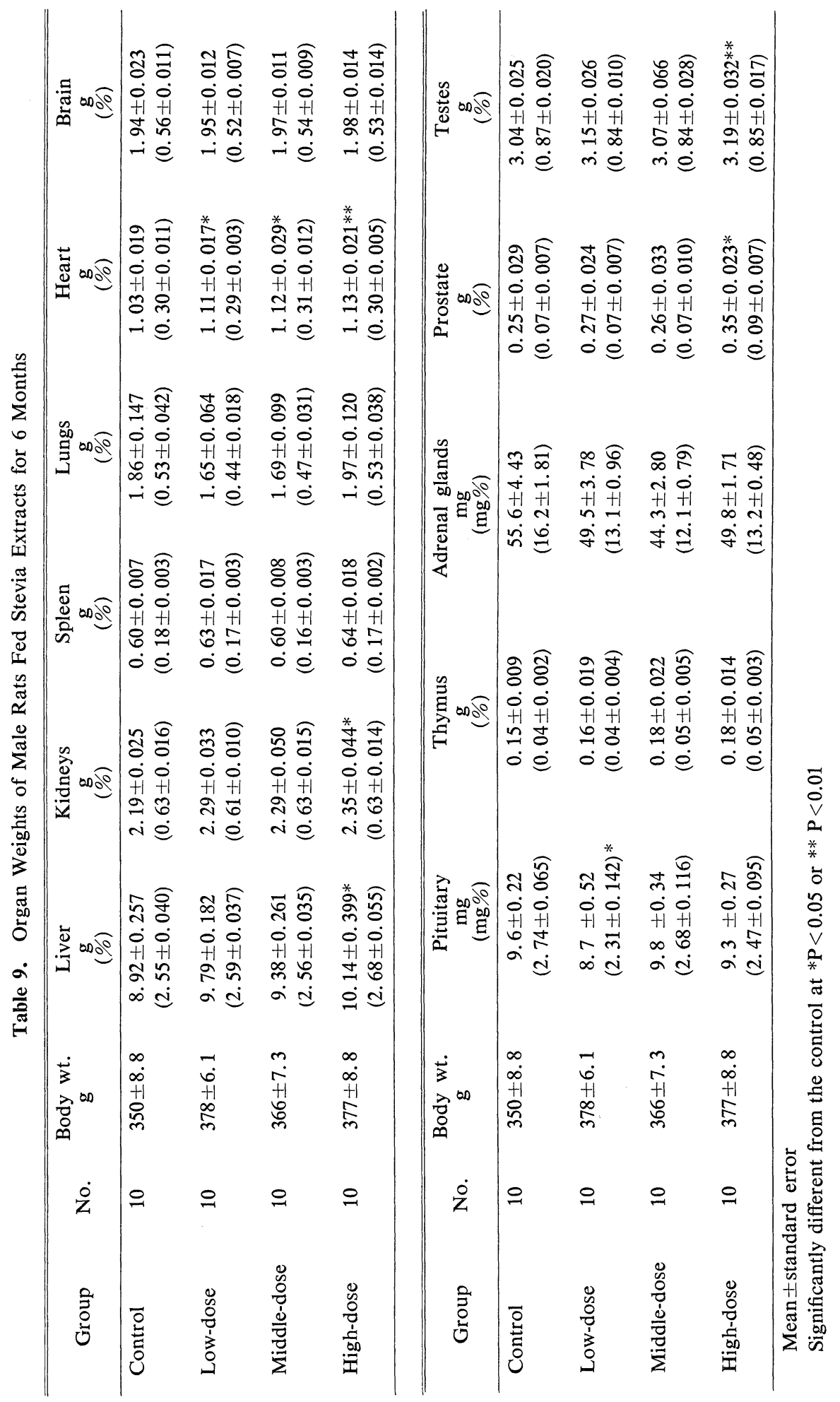




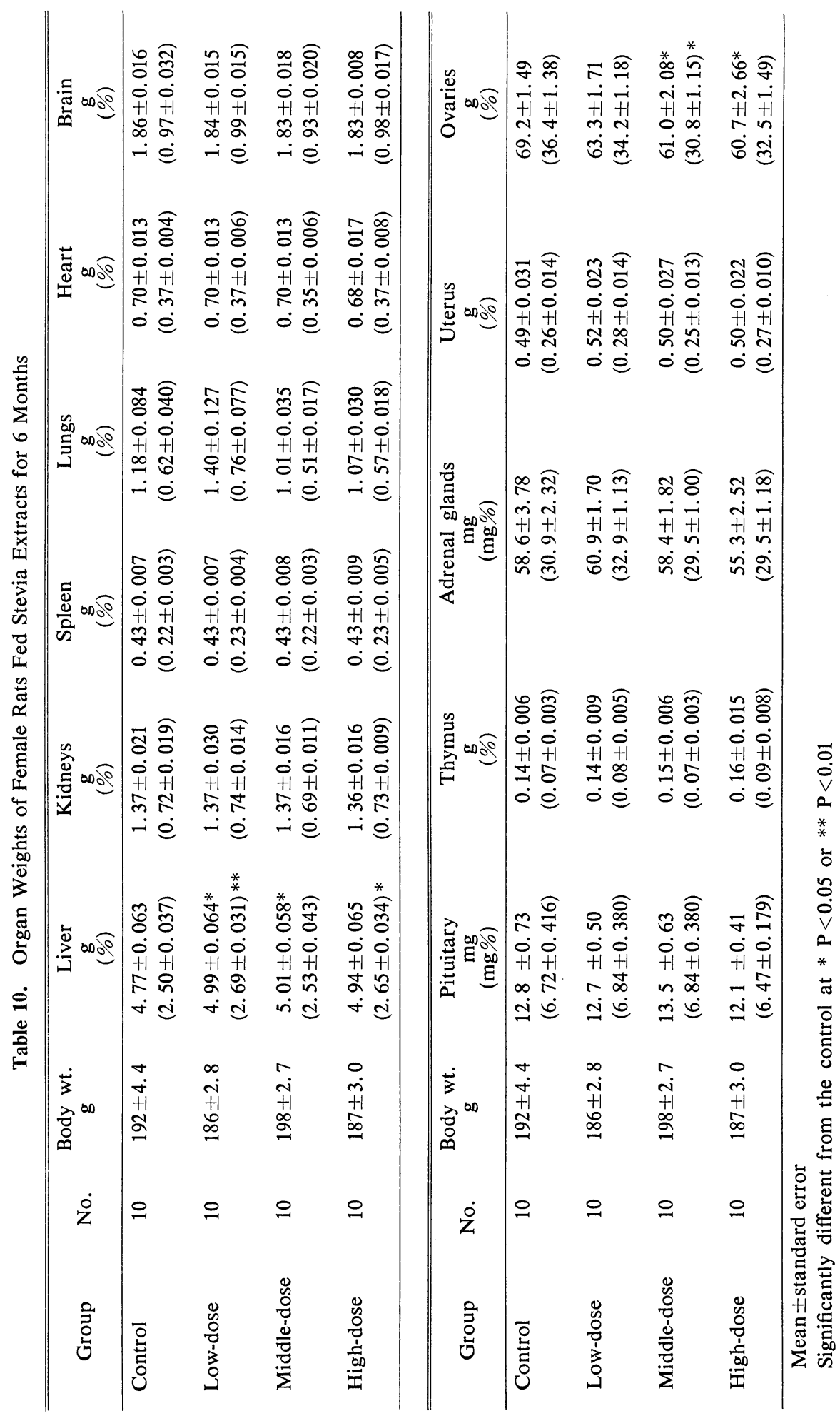




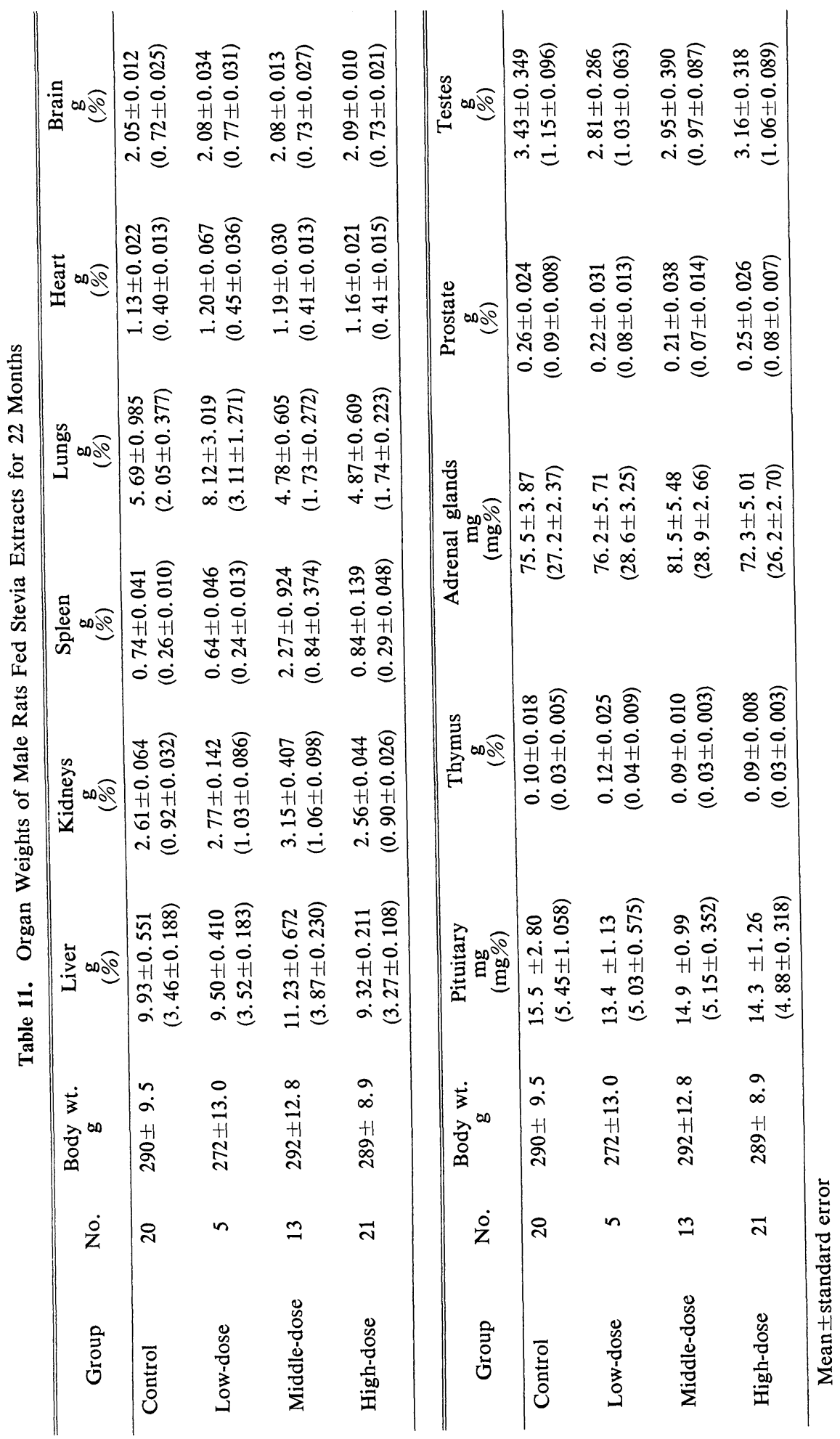




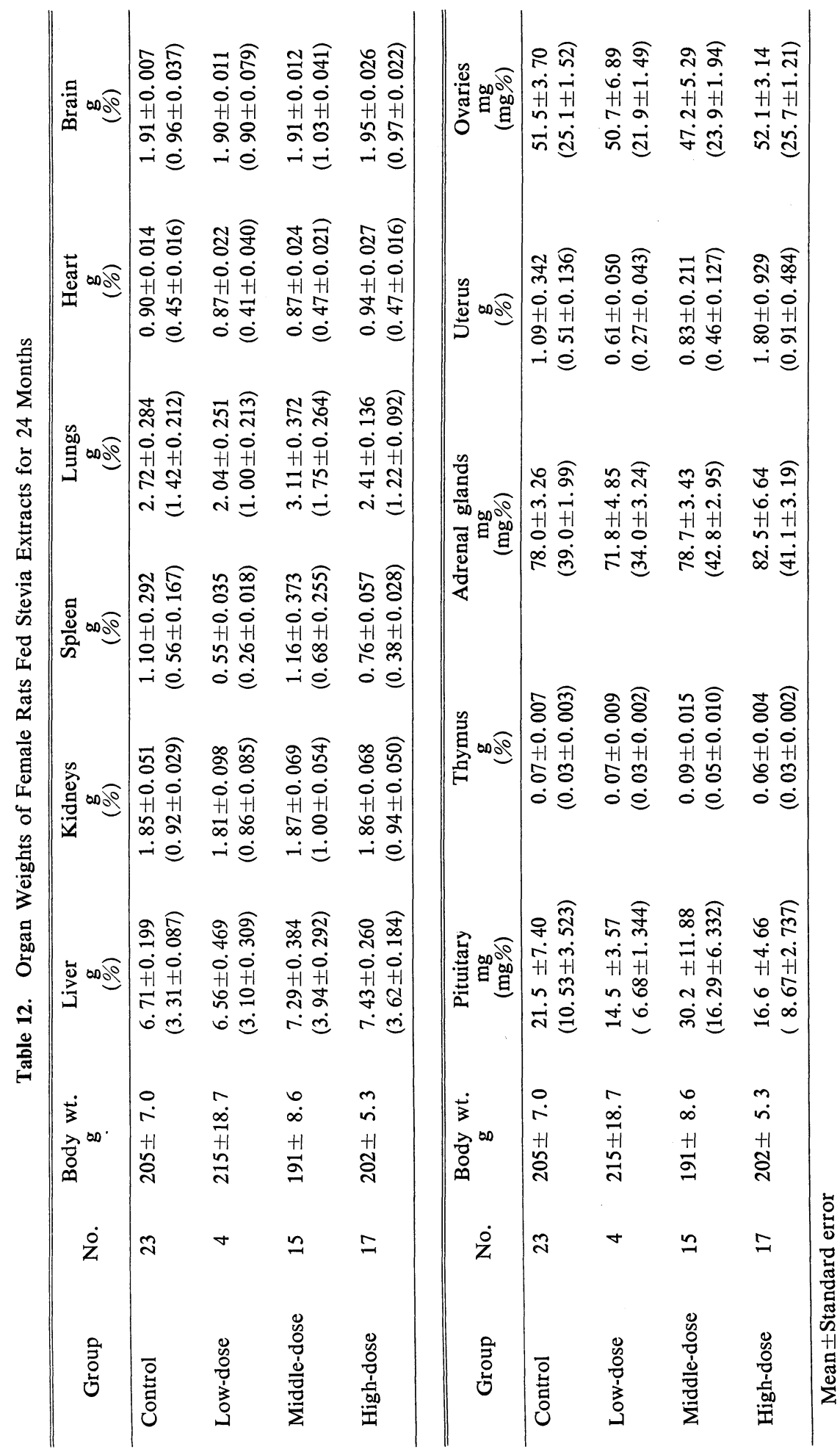


Table 13. Incidence of Non-neoplastic Tissue Changes in Rats Fed Stevia Extracts

\begin{tabular}{|c|c|c|c|c|c|c|c|c|c|}
\hline \multirow[t]{2}{*}{ Organ } & \multirow[t]{2}{*}{ Findings } & \multicolumn{2}{|c|}{$\begin{array}{l}\text { Control } \\
(\mathrm{N}=50)^{*}\end{array}$} & \multicolumn{2}{|c|}{$\begin{array}{l}\text { Low-dose } \\
(\mathrm{N}=10)^{*}\end{array}$} & \multicolumn{2}{|c|}{$\begin{array}{c}\text { Middle- } \\
\text { dose } \\
(\mathbf{N}=50)^{*}\end{array}$} & \multirow{2}{*}{\multicolumn{2}{|c|}{$\begin{array}{l}\text { High-dose } \\
(\mathrm{N}=50)^{*} \\
\mathrm{M}\end{array}$}} \\
\hline & & & $\mathrm{F}$ & & & & $\mathrm{F}$ & & \\
\hline \multirow[t]{3}{*}{ Liver } & Hyperplasia of hepatic bile duct & 11 & 5 & 3 & 2 & 8 & 0 & 6 & 2 \\
\hline & Fatty degeneration of hepatocytes & 4 & 5 & 0 & 3 & 4 & 3 & 4 & 2 \\
\hline & Teratoid nodule & 5 & 10 & 1 & 1 & 5 & 2 & 2 & \\
\hline Esophagus & Excessive cornification & 4 & 9 & - & - & 2 & 4 & 2 & 3 \\
\hline \multirow[t]{3}{*}{ Stomach } & Submucosal fibrosis of forestomach & 7 & 3 & 1 & 2 & 4 & 1 & 0 & 3 \\
\hline & Dilatation of gastric gland cavity & 15 & 22 & 2 & 1 & 12 & 10 & 10 & 12 \\
\hline & Ulcer & 4 & 6 & 1 & 1 & 4 & 2 & 2 & 4 \\
\hline \multirow[t]{2}{*}{ Pancreas } & Hyperplasia of ductules & 2 & 5 & 0 & 2 & 7 & 5 & 2 & 0 \\
\hline & Focal atrophy & 1 & 0 & 0 & 0 & 4 & 5 & 1 & 1 \\
\hline \multirow[t]{5}{*}{ Lung } & Bronchopneumonia & 21 & 13 & 4 & 5 & 23 & 23 & 25 & 17 \\
\hline & Bronchiectatic abscess & 25 & 13 & 6 & 2 & 32 & 19 & 19 & 9 \\
\hline & Bronchitis & 31 & 30 & 9 & 7 & 35 & 37 & 37 & 35 \\
\hline & Congestion & 8 & 4 & 1 & 2 & 8 & 5 & 2 & 3 \\
\hline & Edema & 7 & 4 & 1 & 2 & 8 & 5 & 2 & 3 \\
\hline Trachea & Infiltration of inflammatory cells & 18 & 19 & - & - & 29 & 26 & 20 & 34 \\
\hline \multirow{2}{*}{ Heart } & Focal fibrosis of myocardium & 3 & 1 & 1 & 0 & 2 & 0 & 2 & 0 \\
\hline & Dilatation of heart cavity & 0 & 4 & 1 & 0 & 0 & 1 & 1 & 0 \\
\hline $\begin{array}{l}\text { Coronary } \\
\text { artery }\end{array}$ & Atherosclerosis & 5 & 0 & 1 & 0 & 8 & 0 & 0 & 0 \\
\hline \multirow{2}{*}{$\begin{array}{l}\text { Bone } \\
\text { marrow }\end{array}$} & Increased hematogenesis & 0 & 4 & 2 & 0 & 4 & 1 & 4 & \\
\hline & Decreased hematogenesis & 1 & 0 & 0 & 0 & 1 & 1 & 3 & 3 \\
\hline \multirow[t]{3}{*}{ Spleen } & Pigmentation & 13 & 26 & 1 & 4 & 15 & 30 & 10 & 24 \\
\hline & Increased hematogenesis & 13 & 23 & 4 & 5 & 21 & 24 & 12 & 23 \\
\hline & Atrophy & 7 & 12 & 1 & 1 & 3 & 1 & 1 & 0 \\
\hline $\begin{array}{l}\text { Lymph } \\
\text { nodes }\end{array}$ & Proliferation of plasma cells & 2 & 4 & - & - & 1 & 3 & 8 & 3 \\
\hline \multirow[t]{7}{*}{ Kidneys } & Degeneration of tubular epithelium & 35 & 25 & 8 & 9 & 43 & 37 & 40 & \\
\hline & Regenerative change of tubules & 25 & 23 & 5 & 8 & 21 & 17 & 26 & 16 \\
\hline & Pigmentation of tubular epithelium & 20 & 29 & 1 & 0 & 13 & 8 & 7 & 6 \\
\hline & Calcification & 3 & 5 & 0 & 1 & 0 & 4 & 2 & 4 \\
\hline & Hyaline casts & 45 & 35 & 10 & 10 & 47 & 44 & 45 & 43 \\
\hline & Lymphocyte infiltration & 3 & 9 & 0 & 2 & 7 & 3 & 2 & 0 \\
\hline & Glomerular sclerosis & 6 & 1 & 3 & 3 & 3 & 3 & 4 & \\
\hline \multirow[t]{3}{*}{ Pituitary } & Proliferation of chromophobes & 7 & 13 & 1 & 3 & 4 & 5 & 7 & 11 \\
\hline & Proliferation of blood vessels & 3 & 2 & 2 & 9 & 4 & 9 & 7 & 9 \\
\hline & Pigmentation & 2 & 3 & 1 & 1 & 3 & 3 & 1 & 3 \\
\hline Thyroid & Proliferation of $\mathrm{C}$ cells & 2 & 5 & 0 & 1 & 1 & 5 & 1 & 1 \\
\hline \multirow{4}{*}{$\begin{array}{l}\text { Adrenal } \\
\text { glands }\end{array}$} & Vacuolization of cortical cells & 6 & 18 & 1 & 2 & 16 & 13 & 6 & 6 \\
\hline & Proliferation of medullary cells & 19 & 16 & 3 & 3 & 23 & 13 & 25 & 11 \\
\hline & Dilatation of medullary cells & 16 & 26 & 3 & 8 & 31 & 23 & 19 & 22 \\
\hline & Pigmentation & 4 & 12 & 0 & 0 & 1 & 1 & 2 & 1 \\
\hline Thymus & Atrophy & 20 & 24 & 4 & 8 & 20 & 22 & 19 & 22 \\
\hline \multirow[t]{2}{*}{ Testes } & Decreased spermatogenesis & 34 & - & 7 & - & 39 & - & 37 & - \\
\hline & Proliferation of interstitial cells & 16 & - & 5 & - & 21 & - & 21 & - \\
\hline \multirow[t]{3}{*}{ Uterus } & Abscess & - & 12 & - & 1 & - & 11 & - & 8 \\
\hline & Hyperplasia of endometrium & - & 12 & - & 1 & - & 6 & - & 10 \\
\hline & Dilatation of cavity & - & 9 & - & 1 & - & 5 & - & 7 \\
\hline \multirow[t]{3}{*}{ Ovaries } & Pigmentation & - & 15 & - & 2 & - & 3 & - & 5 \\
\hline & Atrophy & - & 2 & - & 0 & - & 2 & - & 1 \\
\hline & Abscess & - & 2 & - & 0 & - & 2 & - & 1 \\
\hline
\end{tabular}

* The total of rats that died or were sacrificed during the study and those that survived at the end of the study (at 22 months in the males and at 24 months in the females). 

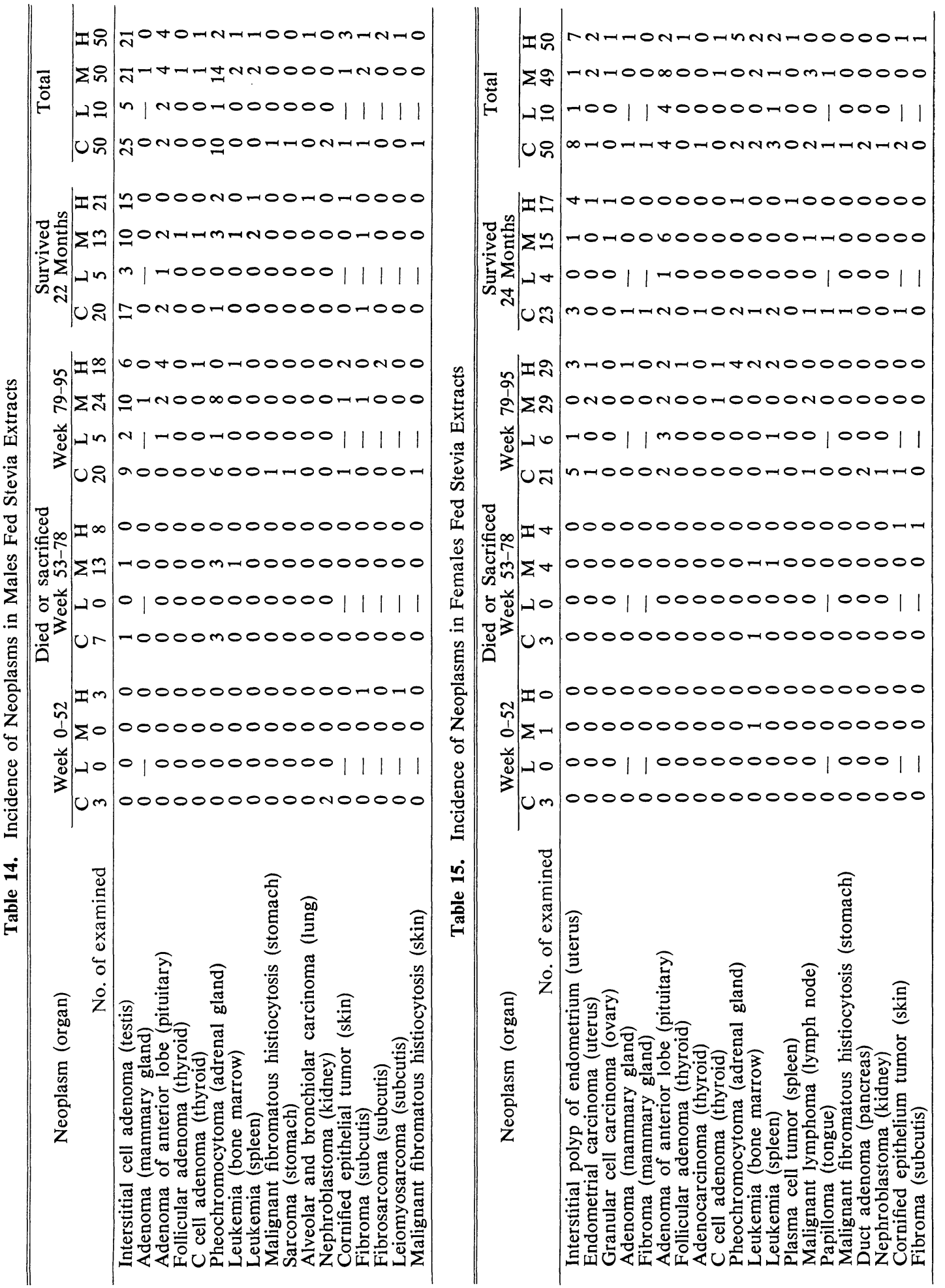
more, which is nearly their lifespan. In this experiment, mortality in the male rats increased rapidly after 18 months, and administration was discontinued at 22 months, when the mortality of the male controls reached $60 \% .{ }^{12)}$ The mortality of the female controls was $54 \%$ at 24 months.

Moribundity and death were caused by rapidly developing tumors, leukemia and exhaustion chiefly owing to pulmonary lesions.

In all groups including the control, the weight of rats tended to decrease after week 69 in males and week 79 in females. This was largely due to aging.

The incidence of proteinuria was significantly higher in the high-dose females at 6 months, but no significant increase of serum urea nitrogen or creatinine was found, nor were pathological changes seen in the kidneys. The incidence of proteinuria was not significantly different at 12 months or at the end of the test.

Although erythrocyte counts in the high-dose group were significantly high in males and significantly low in females at 6 months, there were no significant differences in the hemoglobin and hematocrit values between the controls and the groups fed stevia extracts. Pathological tests did not disclose changes in the hematogenic organs. The decreases of $\mathrm{MCV}$ and $\mathrm{MCH}$ in male rats were secondary effects of increased erythrocyte counts. No significant changes in erythrocyte counts were found at 12 months or at the end of the study.

Blood glucose was increased in both male and female rats given stevia extracts at 6 months. However, urinary glucose was not detected and there were no pathological changes in the liver, pancreas and endocrine organs.

At 6 months, decreases in GOT, GPT and triglycerides were found in female rats fed stevia extracts, but these changes were not obviously related to clinical disorders.

The absolute weights of the liver, kidneys, heart, prostate and testes were increased in male rats, and the absolute weight of the ovaries was decreased in female rats, at 6 months in a doserelated manner. The relative weights of these organs, however, were not significantly different. No differences in organ weights were observed at 12 months or at the end of the study.
Enlargement of the spleen in both sexes was found in some of the rats sacrificed during the study or at the end of the study; no male and 7 females of the controls, 1 male and 2 females in the low-dose group, 3 males and 9 females in the middle-dose group, and 4 males and 10 females in the high-dose group. Some of the enlarged spleens had a pale-yellowish appearance with histological changes and the rats had high leucocyte counts in the blood. Therefore, we diagnosed them as having leukemia, a commonly-found disorder in aged rats. Although it was not observed in male controls, enlargement of the spleen was found in female controls and the incidence in females was not significantly larger in groups fed stevia extracts than that in the controls. Consequently, we think that the finding was probably not related to the feeding with stevia extracts.

There were a variety of changes in various organs. In most cases, the incidences of the various non-neoplastic lesions in rats given stevia extracts and in the controls were similar. Most of the non-neoplastic changes are lesions found commonly in aged rats, ${ }^{13), 14)}$ and we think that they were unrelated to the administration of stevia extracts.

Various types of neoplasms were observed in several different organs in rats of both sexes. Here, too, almost all were of types that are sometimes found in aged rats of F344 strain. ${ }^{15) \sim 17}$ Other neoplasms were found only in the controls. We concluded that these neoplasms were not caused by the administration of stevia extracts.

Although more than half of the rats of both sexes died during the test period, at the 18th month the mortalities in the controls were $20 \%$ for males and $12 \%$ for females. Furthermore, organs of all rats were observed macroscopically and microscopically. Consequently, we think that this experiment should have uncovered any significant chronic toxicological features of stevia extracts.

In conclusion, when male and female F344 rats were fed stevia extracts at levels up to $1 \%$ of the weight of their feed for about two years, no significant dose-related changes were found in growth, general appearance, hematological and blood biochemical findings, organ weights, 
or macroscopic and microscopic findings. We consider that the highest dose causing no effect in rats is $550 \mathrm{mg} / \mathrm{kg}$ of stevia extracts under the conditions of these experiments.

\section{Acknowledgement}

The authors wish to thank Dr. Makoto Enomoto for his skillful accomplishment of anatomical diagnosis.

\section{References}

1) Akashi, H., Yokoyama, S.: Shokuhin Kogyo (The Food Industry) 18 (20), 34 43 (1975).

2) Planas, G. M., Kuć, J.: Science 162, 1007 (1968).

3) Mori. N., Sakagami, M., Takeuchi, M., Shinpo, K., Tanabe, T.: J. Food Hyg. Soc. Japan 22, 409 414 (1981).

4) Okumura, M., Fujita, Y., Imamura, M., Aikawa, K.: ibid., 19, 486 490 (1978).

5) Ishidate, Jr., M., Yoshikawa, K., Sofuni, T.: Hen'igen to Dokusei (Mutagens and Toxicology) No. 12, 82 90 (1980).

6) Ishidate, Jr., M., Sofuni, T., Yoshikawa, K., Hayashi, M., Nohmi, T.: Food Chem. Toxicol. 22, $623 \sim 636$ (1984).

7) Boeki Nichinichi Tsushinsha: "Togyo Nenkan 1983 1984" (Yearbook for Sugar Industry in
Japan) p. $248 \sim 249$ (1983), Boeki Nichinichi Tsushinsha, Tokyo.

8) Yamazaki, M., Noguchi, Y., Tanda, M., Shintani, S.: J. Takeda Res. Lab. 40, 163 187 (1981).

9) Dunnett, C. W.: J. Am. Statist. Assoc. 50, 1096 1121 (1955).

10) Dunnett, C. W.: Biometrics 20, 482 491 (1964).

11) Scheffé, H.: Biometrika 40, 87 104 (1953).

12) United States Pharmaceutical Manufacturers' Association Guidelines for the Assessment of Drug and Medical Device Safety in Animals (1977); cited from Ohmori, Y. (supervised): "OECD Guidelines for Toxicology Testing of Chemicals”, p. 207 (1981). Kagaku Kogyo Nipposha, Tokyo.

13) Snell, K. C.: "The Pathology of Laboratory Animals" (Ribelin, W. E., McCoy, J. R., ed.) p. $241 \sim 302$ (1965), C. C. Thomas, Illinois.

14) Nishibe, T.: J. Nara Med. Ass. 27, $1 \sim 26$ (1976).

15) Maekawa, A., Kurokawa, Y., Takahashi, M., Kokubo, T., Ogiu, T., Onodera, H., Tanigawa, H., Ohno, Y., Furukawa, F., Hayashi, Y.: Gann 74, 365 372 (1983).

16) Sass, B., Rabstein, L.S., Madison, R., Nims, R. M., Peters, R. L., Kelloff, G. J.: J. Natl. Cancer Inst. 54, 1449 1456 (1975).

17) Jacobs, B. B., Husbey, R. A.: ibid., 39, 303 307 (1967). 